\title{
Attitude information collection and transmission of three-component geophone and positioning system
}

\author{
Tailang Zhao *, Dongyang Liu, Lin Liu, Han Niu \\ (State Key Laboratory of Coal Resources and Safe Mining ,China University of Mining \&Technology ,Beijing ,100083,China ) \\ 865514332@qq.com
}

\begin{abstract}
In actual construction, there existing a big error in GPS, the geophones embedded non-vertical, azimuth has some deviation.However, the position information of shot point and receiver point , and the azimuthal angle information of 3D3C geophones determine the accuracy of seismic exploration final interpretation. While through orientation and coordinate data collection, loading the observation system information and geophone attitude information which stored in data receiving unit to the seismic data .After $R-T$ rotation, we obtain the common-geophone seismic gather, obviously the continuity of the lineups are better.
\end{abstract}

Keywords—attitude information; 3D3Cgeophone;positioning system

\section{INTRODUCTION}

With the development of seismic exploration, 3D3C seismic collection has been viewed by explorers as a new technique and a new method. While in actual construction, there existing a big error (5 to 10 meters)in GPS, the geophones embedded non-vertical , azimuth has some deviation.However, the position information of shot point and receiver point , and the azimuthal angle information of 3D3C geophones determine the accuracy of seismic exploration final interpretation .In this paper, we use UWB positioning system to make precise positioning of shot point and receiver point positions. Meanwhile, we use 3D3C geophones attitude information collecting and transmission system to realize the accuracy record of azimuthal angle and dip angle information .

\section{THEORY AND METHOD}

\section{A. Positioning Principles}

Signal will decay with distance increased when propagating in the space, thus the signal received by signature tag must be some distance-based function .If transmitting power of the base station and the channel environment between base station and signature tag are known,we can stimulate the function of signal strength and distance.Around every positioning base station formed round and round signal equal strength lines .If combining the equal strength lines of three base stations, we can confirm the detail of location tag position .

The relationship between signal strength and distance in space is :

$$
\mathrm{P}_{\mathrm{r}}(d)=P_{t} G_{t} G_{r}\left(\frac{\tau}{4 \pi d}\right)^{2}
$$

Where $\mathrm{Pt}$ is transmitting power, $\mathrm{Gt}$ is transmitting terminal gain , $\mathrm{Gr}$ is receiving terminal gain ,these are certain amounts in a known system.$\tau$ is path loss exponent, related to specific channels d is the distance need to be estimated .

\section{Azimuth Transform Principle}

In multiwave seismic acquisition 3D observation system ,the geophones arranged along in-line(inline, $\mathrm{x}$ ), Generally not through the shot point and not agree with radial component (radial, $\mathrm{R}$,offset direction ); accordingly cross-line is not agree with normal direction (transverse, T). Thus rotating coordinate in order to obtain radial horizontal component that we need when processing .

The formula below is how to transform the collecting coordinate $\mathrm{x}-\mathrm{y}$ to coordinate R-T :

Rotation formula in two-dimension is :

$$
\left[\begin{array}{l}
R \\
T
\end{array}\right]=\left[\begin{array}{ll}
\cos \theta & \sin \theta \\
-\sin \theta & \cos \theta
\end{array}\right]\left[\begin{array}{l}
x \\
y
\end{array}\right]
$$

Rotation formula in three-dimension is :

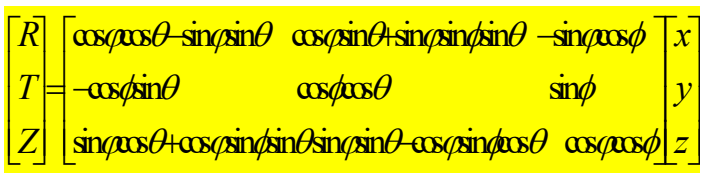

Where $\mathrm{R}$ is radial component, the component of offset direction. $\mathrm{T}$ is normal component, perpendicular to offset direction component. $\mathrm{Z}$ is $\mathrm{Z}$-axis component after rotation. $\mathrm{X}$ is in-line data received by geophone $y$ is cross-line data received by geophone . $\mathrm{z}$ is $\mathrm{z}$-axis data received by geophone.$\theta$ is the angle which $Z$ rotated around $z$-axis.$\Phi$ is the angle which $\mathrm{R}$ rotated around $\mathrm{x}$-axis.$\varphi$ is the angle which $\mathrm{T}$ rotated around $\mathrm{y}$-axis.

Workflow

Acquisition Operation Positioning System

- Establish collection area:using multi area positioning UWB sensor unit (at least 3)to establish collection area.

- $\quad$ Equip the UWB tag unit :equipping the UWB tag 
unit on collecting facility .

- $\quad$ Facility positioning and information record : when finish setting UWB sensor unit and UWB tag unit, the main control unit can be networking and positioning .

B. 3C Geophone Attitude Information Collection and Transmission System

(1)Prepare apparatus,the three-component geophone in collection system has a three-dimensional electronic compass which has wireless positioning module and wireless transmission module. The wireless transmission base station receives the wireless signal in collection system and connected with data receiving unit terminal computer and data center. The positioning base stations conclude main control base station and assistant base station in collection system ,the main control positioning base station connect with router ,data center ,positioning system management platform through network cable .

(2)According to the observation system ,embed the 3C geophone with $3 \mathrm{D}$ electronic compass.Confirming the spatial location of electronic compass through the positioning software in positioning system ,corresponding with geophone line-stack mark, meanwhile, establishing the point-to-point transmission agreement of electronic compass.

Establishing wireless transmission network through wireless signal transmission base station,under the effect of the control center, we set up a separate agreement on every geophone compass wireless sensor module,completing pointto-point transmission, confirming the orientation information which transmitted to the terminal is right corresponding to the geophone .

When finishing setting up the collection system .before every time collecting the seismic data,we all start the positioning program to collect the spatial position information of electronic compass and corresponding with the line and stake mark of geophone ,preparing for point-topoint transmission ,according to the relationship between compass location information and geophone line-stake mark ,we encode the compass data information,through point-to-point transmission storing in data receiving unit, completing one information collecting program.

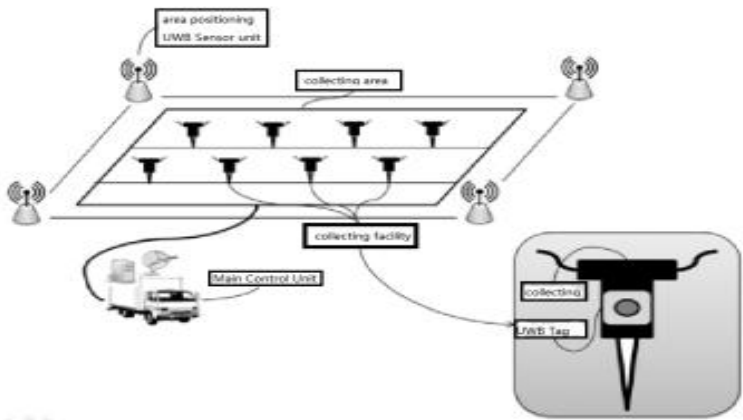

Fig.1 Positioning system implement block diagram

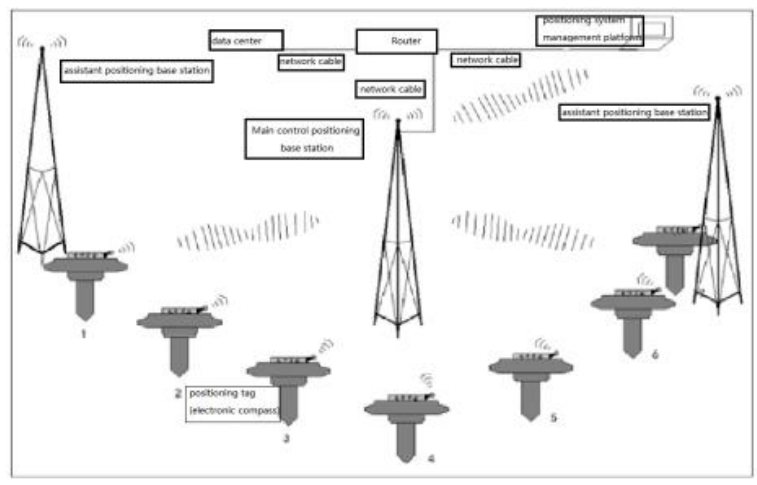

Fig.2 Orientation information collection system implement block diagram

\section{FIELD EXAMPLE}

In actual construction, there are some error between real azimuth and theoretical azimuth (according to coordinate),so after R-T rotation ,the continuity of common-geophone seismic gather lineups that obtained is bad .,shows in figure 3. While through orientation and coordinate data collection ,loading the observation system information and geophone attitude information which stored in data receiving unit to the seismic data .After R-T rotation,we obtain the common-geophone seismic gather ,shows in figure 4 ,obviously the continuity of the lineups are better.

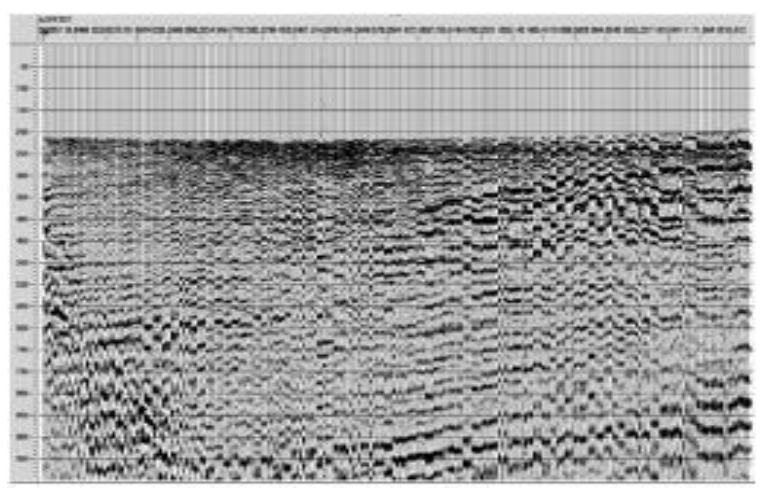

Fig.3 seismic profile without attitude processing

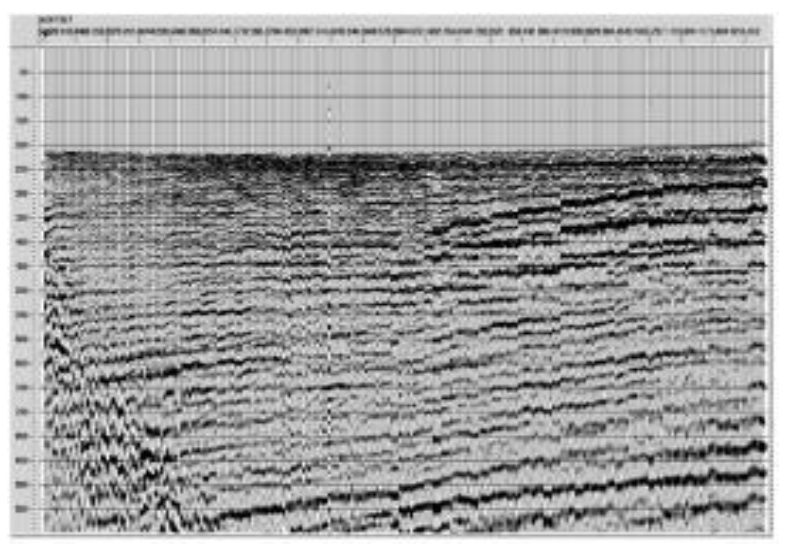

Figure 4 seismic profile processed with attitude information 


\section{CONCLUSION}

With the development of seismic exploration, 3D3C seismic collection has been viewed by explorers as a new technique and a new method. While in actual construction ,there existing a big error in GPS, the geophones embedded non-vertical ,azimuth has some deviation.However , the position information of shot point and receiver point , and the azimuthal angle information of 3D3C geophones determine the accuracy of seismic exploration final interpretation. While through orientation and coordinate data collection ,loading the observation system information and geophone attitude information which stored in data receiving unit to the seismic data .After R-T rotation ,we obtain the common-geophone seismic gather , obviously the continuity of the lineups are better.

\section{REFERENCES}

[1] Du,W.F.,Peng,S.P.,Gou.J.W.,2015,Design and evaluation of converted wave acquisition geometry for coalfield seismic exploration:Journal Of China Coal Society,40(6),1428-1434.

[2] He.D.K.,2011,Key Techniques Analysis about Acquisition Planning and Data Processing in the Multi-Wave Seismic Propecting: China university of mining and technology. 